\title{
Chemical exchange between the protocore and the (basal) magma ocean
}

\section{REIDAR G TRONNES}

\author{
CEED-NHM, Univ. of Oslo
}

Presenting Author: rtronnes@uio.no

The Earth-Theia collision was sufficiently energetic to melt and partly vapourise the silicate part of the protoplanets [1] and emulsify and mix much of the core metal into the silicate melt. The subsequent exothermal separation and sinking of metal droplets in the peridotitic magma ocean (MO) would have occurred at high supra-liquidus temperatures, e.g. $5600 \mathrm{~K}$ [2], at core-mantle boundary (CMB) pressure conditions. High temperatures drive the exhange equilibrium: $2 \mathrm{Fe}^{\text {metal }}+\mathrm{SiO}_{2}$ silicate $=\mathrm{Si}^{\text {met }}+2 \mathrm{FeO}^{\text {sil }}$ towards the products, resulting in a peridotitic magma ocean with low $\mathrm{Si} /(\mathrm{Mg}+\mathrm{Fe})$ and $\mathrm{Mg} / \mathrm{Fe}$ ratios [2-6]. Planetary cooling from above reverses the equilibrium, segregating more Fe-rich metal in the silicate liquid, where the $\mathrm{Si} /(\mathrm{Mg}+\mathrm{Fe})$ and $\mathrm{Mg} / \mathrm{Fe}$ ratios increase. The thermal gradient, resulting in denser FeO-rich liquid with increasing depth, combined with the sinking of metal drops, would inhibit convective overturn, increasing the thermal gradient [2]. This effect, along with the strongly elevated $\mathrm{Mg} / \mathrm{Fe}$ ratio and correspondingly low density of bridgmanite $(\mathrm{bm})$ relative to coexisting silicate melt [6,7], leads to early bm crystallisation at a neutral buoyancy level, probably within the $1500-2000 \mathrm{~km}$ depth range $[6,8]$. The MO above the neutral buoyancy level would solidify rapidly (5-100 My), leaving a long-lived basal $\mathrm{MO}(\mathrm{BMO})$ above the CMB. With further cooling the core-BMO exchange of $\mathrm{SiO}_{2}$ and $\mathrm{FeO}$ would "buffer" the $\mathrm{Si} /(\mathrm{Mg}+\mathrm{Fe})$ and $\mathrm{Mg} / \mathrm{Fe} \mathrm{BMO}$ ratios at high levels, promomoting and prolonging the bm-precipitation near the BMO ceiling and supressing ferropericlase crystallisation. The solidified lower mantle is likely to preserve convectively aggregated, refractory and viscous bm-domains in the 1000-2500 $\mathrm{km}$ depth range $[6,9]$. Such domains, confined to ring-like structures peripheral to the broad outflow (upwelling) colums above the LLSVPs in the D"zone, may stabilise the degree- 2 convection pattern and source bm with primordial-like He-isotope ratios.

[1] Lock et al. 2018 JGR [2] Laneuville et al. 2018 PEPI [3] Malavergne et al 2004 GCA [4] Tsuno et al. 2013 GRL [5] Hirose et al. 2017 Nat. [6] Trønnes et al. 2019 Tectonophys. [7] Tateno et al. 2014 JGR [8] Petitgirard et al. 2015 PNAS [9] Ballmer et al 2017 Nat. Geosci. 